\title{
Virtual Reality for Sensorimotor Rehabilitation Post-Stroke: The Promise and Current State of the Field
}

\author{
Gerard G. Fluet · Judith E. Deutsch
}

Published online: 5 February 2013

(c) Springer Science + Business Media New York 2013

\begin{abstract}
Developments over the past 2 years in virtual reality (VR) augmented sensorimotor rehabilitation of upper limb use and gait post-stroke were reviewed. Studies were included if they evaluated comparative efficacy between VR and standard of care, and or differences in VR delivery methods; and were CEBM (center for evidence based medicine) level 2 or higher. Eight upper limb and two gait studies were included and described using the following categories hardware (input and output), software (virtual task and feedback and presentation) intervention (progression and dose), and outcomes. Trends in the field were commented on, gaps in knowledge identified, and areas of future research and translation of VR to practice were suggested.
\end{abstract}

Keywords Stroke - Rehabilitation - Virtual reality . Virtual environment $\cdot$ Gait $\cdot$ Walking $\cdot$ Mobility $\cdot$ Balance $\cdot$ Upper extremity $\cdot$ Arm $\cdot$ Hand $\cdot$ Robotics $\cdot$ Haptics . Immersive $\cdot$ Semi-immersive

\section{Introduction}

The introduction of virtual reality (VR) augmented sensorimotor rehabilitation was heralded as a therapy that

\section{G. G. Fluet}

Department of Rehabilitation and Movement Science, University of Medicine and Dentistry of New Jersey, 65 Bergen Street, Newark, NJ 07101, USA

\section{J. E. Deutsch $(\square)$}

Department of Rehabilitation and Movement Science, Rivers Lab, University of Medicine and Dentistry of New Jersey, 65 Bergen Street, Newark, NJ 07101, USA

e-mail: deutsch@umdnj.edu promised ecologically valid, intensive task specific training [1]. It was purported to provide multi-sensory training that would transfer from the virtual world to the real world [2]. Additionally, it was suggested that VR could deliver training intensity (repetitions and duration) associated with neuroplasticity and positive behavioral adaptations [3] because it was particularly well suited to very high training doses $[4,5]$. Early reviews of the field have shown that promise to be partially met $[6,7]$. Virtual reality technology and its application to motor rehabilitation have been described elsewhere $[5,8]$ and will not be the focus of this paper.

The study of virtual reality-enhanced rehabilitation has a short life of approximately a decade. The field has matured. There are two alternating bi-annual international conferences, International Conference on Virtual Reality Rehabilitation http://virtual-rehab.org/2013/ (originally the Workshop on Virtual Reality originated by Drs. Burdea and Thalman) and the International Conference on Disability Virtual Reality and Associated Technologies www. icdvrat.reading.ac.uk/ (organized by Dr. Sharkey). Both of these meetings publish proceedings. Recently members of both groups have formed the International Society for Virtual Reality (www.isvr.org).

Work in the field has merged engineering, cognitive neuroscience, biomechanics and rehabilitation sciences. There is an arc from development to validation and subsequent efficacy testing. Early in the field's development papers were primarily technical with single case reports and descriptive studies $[9,10]$. The first randomized controlled trial on walking recovery was published in 2004 [11] and the field's progress was reviewed with a focus on upper limb (UL) rehabilitation [12], more globally [4], with emphasis on video capture systems [13] and in a Cochrane review [7•]. The purpose of this paper is to review and 
comment on the randomized controlled trials (RCTs) published since the stroke and on virtual reality Cochrane review [7•], with an emphasis on evidence of VR technologies' efficacy for sensorimotor rehabilitation of the upper limb and gait post-stroke, and translation to practice. Further, we will speculate on research to advance the science of the field and future directions for the integration of VR in stroke rehabilitation.

\section{Method}

A Medline search using the terms "virtual reality" and "stroke" for the period of March 2010 to the present was performed. This time period was selected to include articles that were more recent than those examined in stroke and virtual reality Cochrane review [7•]. Both authors independently reviewed the citations and selected articles that met the following criteria: were RCTs that compared standard of care to virtual reality, or compared different delivery methods in VR. The inclusion criteria of the latter, distinguishes this paper from the recent Cochrane review. Studies were excluded if they did not meet the CEBM level of evidence three or higher (http://www.cebm.net/), had a mechanistic or validation of technology focus or used offthe-shelf video games. Video games are often grouped with virtual reality studies, but for purposes of this paper they were excluded. Agreement between independent reviews was determined by consensus.

The search yielded 50 articles of which ten met the inclusion criteria. Information from the articles was extracted using the following categories:

\section{Sample Number of participants, time post-stroke (in months), motor function and cognition. \\ Hardware (1) Interface collecting movement data (input): such as a camera, sensorized glove or robotic exoskeleton, from the subject.}

(2) Equipment presenting (output)

(a) Visual information: such as a computer screen or a head mounted display, and, or,

(b) Tactile information (which is used to augment the visual and auditory stimuli, presented with haptics, (such as an exoskeleton) and, or, real contact (such as mixed reality systems interacting with real objects) to the subject.

Simulation (1) The virtual task that participants perform.

(2) The feedback provided to augment motor learning: (a) knowledge of results (KR) which is information related to achievement of the movement goal and (b) Knowledge of Performance (KP) which is information about subject's movement strategy [14].

(3) The visual presentation either in two or three dimensions and perspective first or third person.
Comparison Experimental (typically VR) and control conditions (either standard of care or alternate form to deliver VR).

Intervention (1) Dose: the number of minutes, days and weeks of the intervention.

(2) Progression: the method used to increase training difficulty Treatment progression was achieved with combinations of hardware, software and clinician inputs. Exercise progression was driven by algorithms, which were implemented in the software or performance information generated by the software, which was then used by clinician to make decisions. Inputs for the algorithms were attributes of motor performance such as speed, accuracy, trajectories, goal attainment, which in turn manipulated the physical properties of the interaction such as: weight, size, speed of objects in a simulation.

Outcomes Pre-Post testing for all studies and follow-up if available.

Authors independently extracted the data for the UL (GF) and gait studies (JD).

\section{Results}

Since the Cochrane review on stroke and virtual reality was published, there have been ten more RCTs on the topic with the majority of these studies addressing rehabilitation of the UL (8) compared to gait and mobility (2). The findings are presented in Tables 1: UL studies comparing VR to standard of care [15••, 16, 17, 18•], Table 2: UL studies comparing VR presentation [19, 20•, 21••, 22], and Table 3: gait studies [23, 24•].

\section{Discussion}

In the Cochrane review there were eight studies on the UL and three on gait and mobility. The main finding of that review was the evidence to support the use of VR over standard of care for UL but not gait rehabilitation poststroke. In this paper we look at comparative efficacy as well, but also at important aspects of technology delivery. It is noteworthy that the Cochrane review covered a period of six years and this paper represents only two-and-a-halfyear period. The quantity of the clinical trials in VR research on comparative efficacy and technology delivery continues to progress with a disproportionately larger number of studies on the UL. To provide a perspective on the field, we discuss and comment on each of the elements in the results tables. 


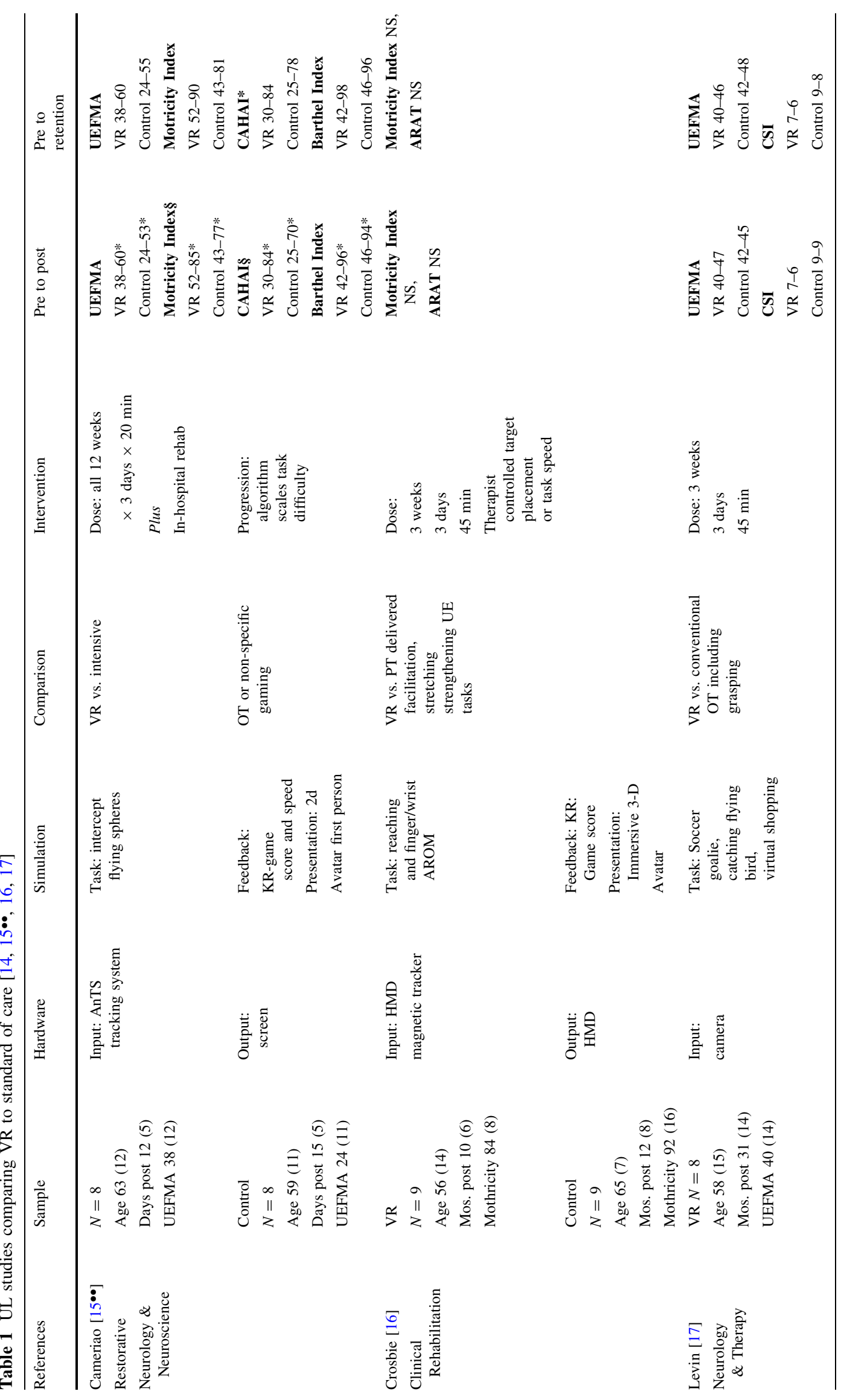




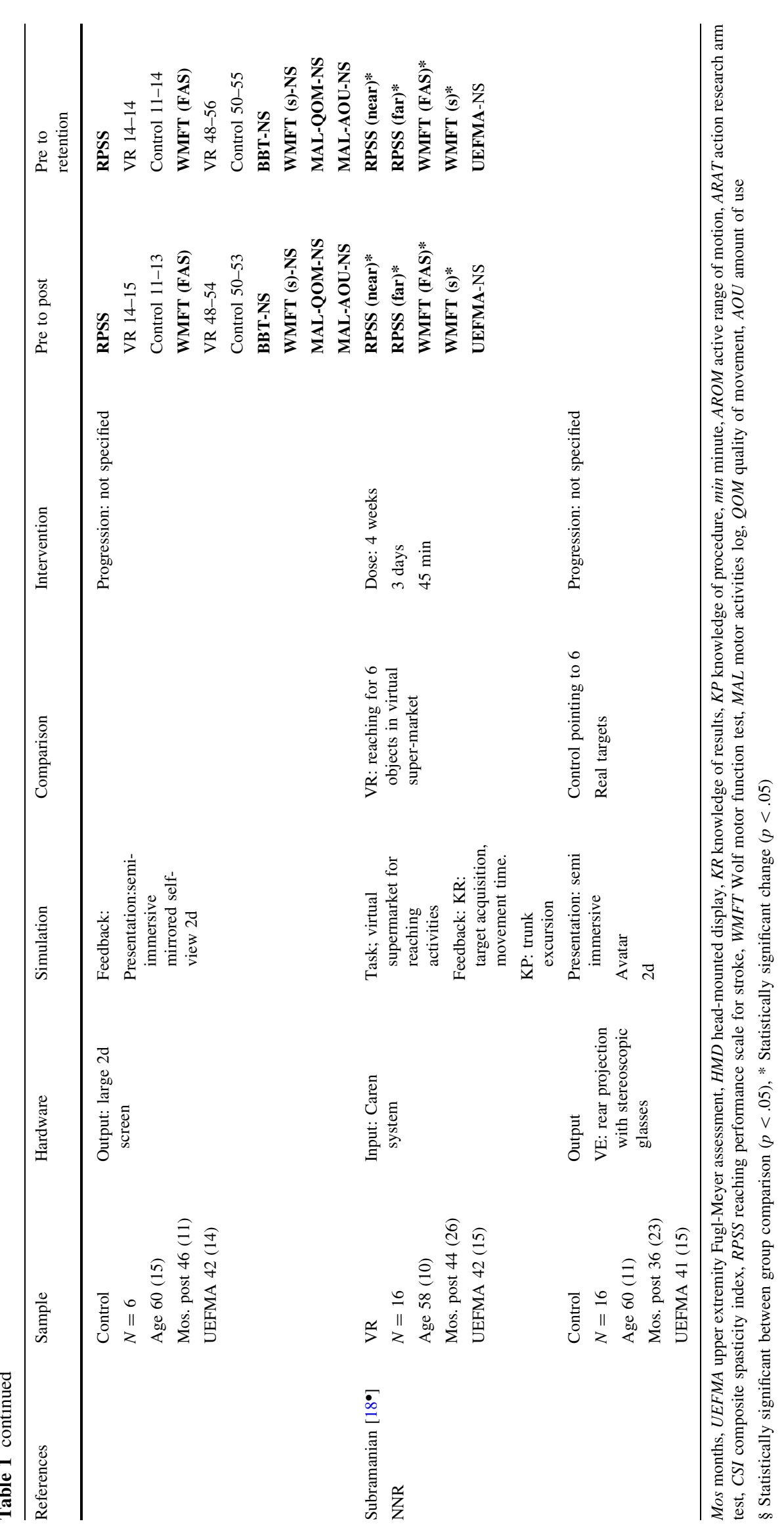




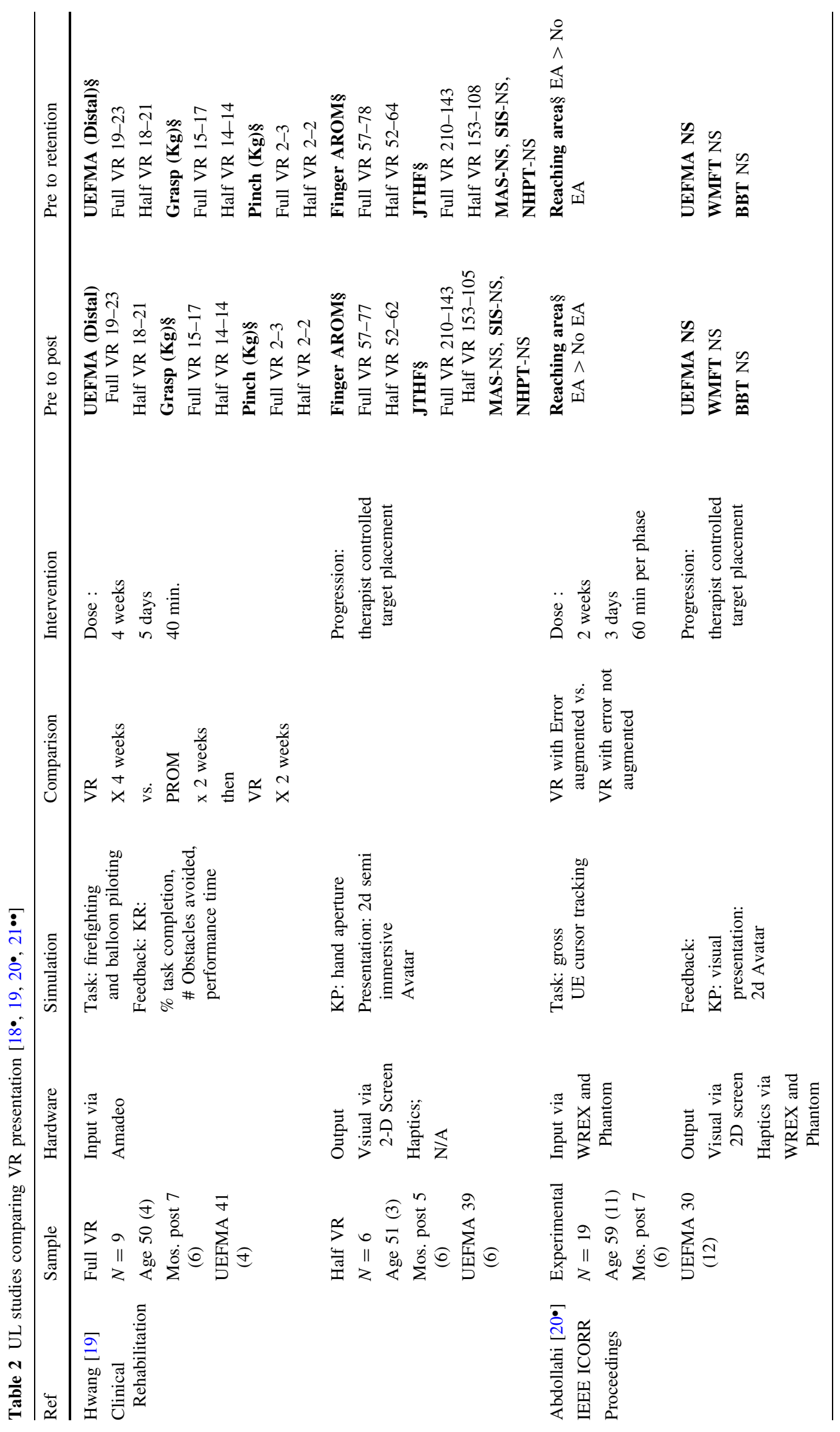




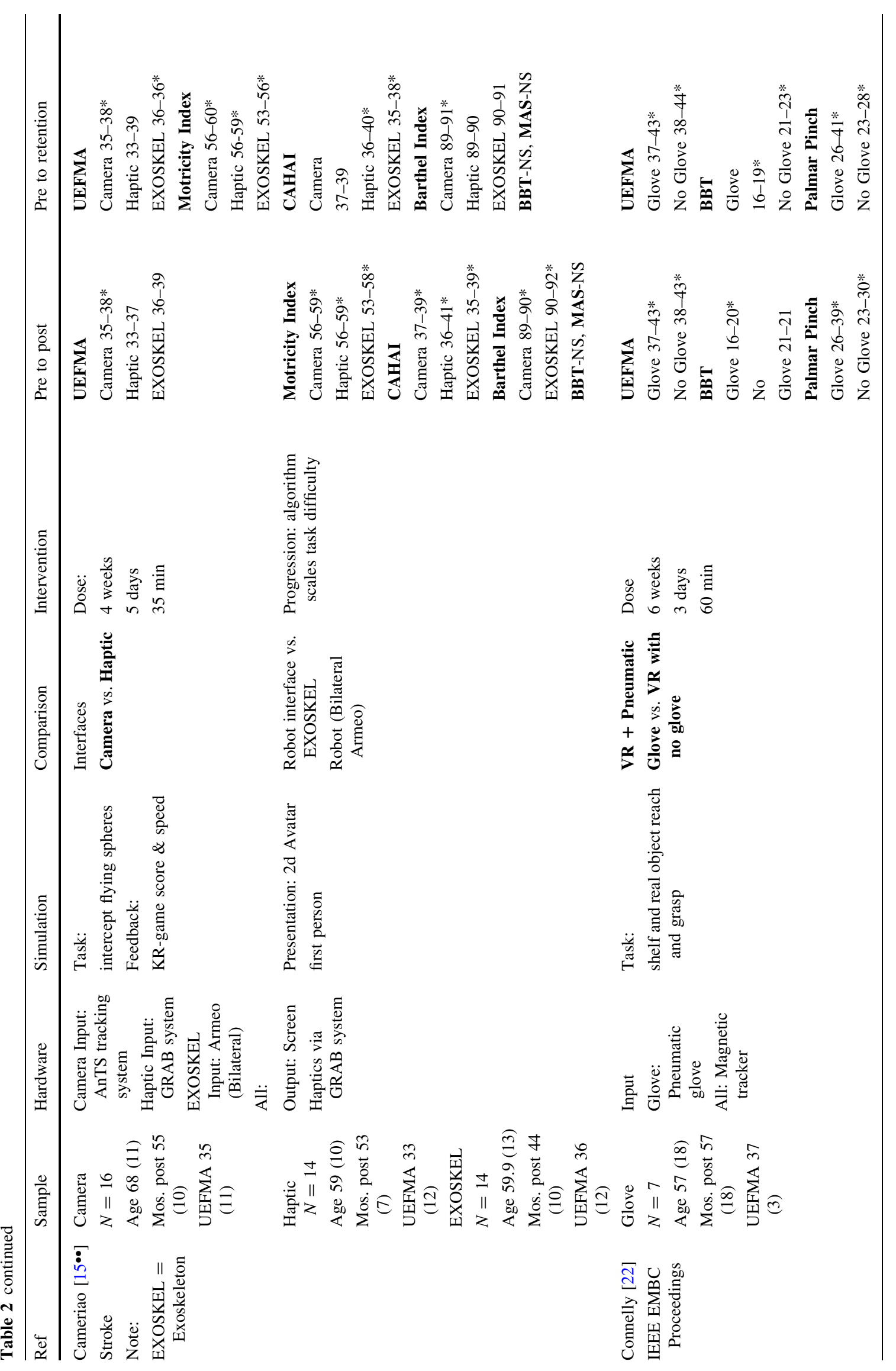




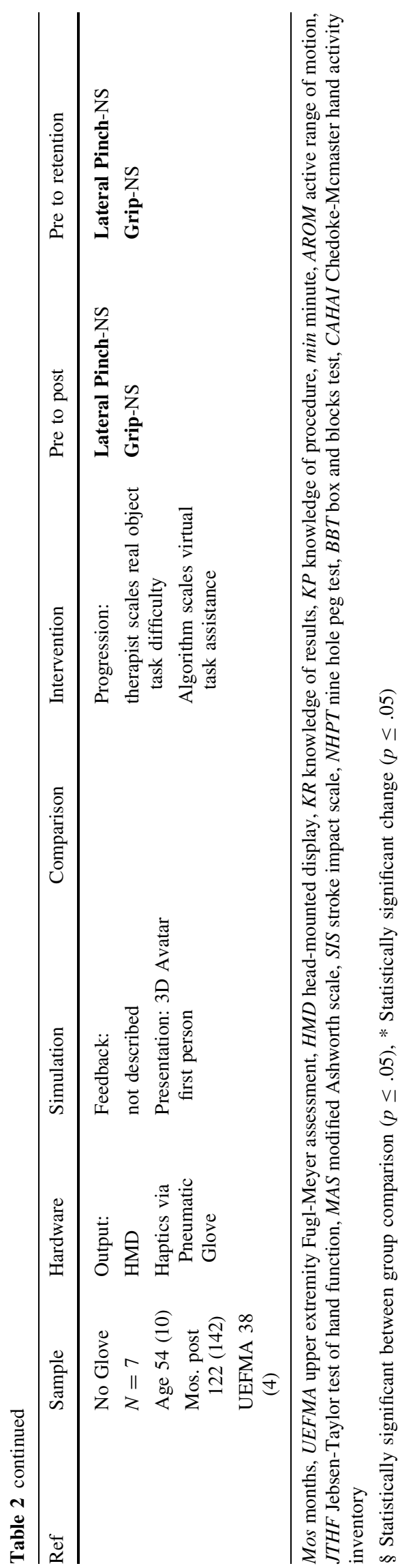

The participants in the studies were typically in the chronic phase (greater than 6 months) post-stroke. The exceptions were Cameriao [15••], who studied acute (4-22 days post) participants in the inpatient setting, and Hwang [19] and Levin [17], who studied sub-acute participants (both less than 3 months).

Cognitive and perceptual characteristics of the participants were mentioned in all studies. Standardized measures of cognition were presented in four studies [16, 17, 18•, 19] but none characterized the cognitive abilities of their sample. Only two studies described a cut-off score on a validated measure of sensory function or perception: Hwang [19], who used the Nottingham Sensory Scale [25], and Crosbie [16], who utilized the mental test [26]. However, neither described their participants' cognitive, perceptual and sensory abilities. The apparent assumption that cognition and perception have an impact on the ability to perform virtual rehabilitation activities is demonstrated by their use as participation criteria in all of these studies. Future characterization of the cognitive, sensory and perceptual abilities of the subjects participating in trials will increase both the external validity and targeted application of VR. It will also reduce the artificial separation between cognitive and motor rehabilitation. Some of this work is already taking place by using VR to address both cognitive and motor impairments for people with PD [27•] and acquired brain injury [28].

Most participants in both UL and gait studies had moderately severe motor control impairments. For the UL studies, moderate severity was measured with scores between 30 and 42 out of 44 points in the upper extremity Fugl Meyer assessment (UEFMA) [29], representing active movements characterized by synergy and minimal manipulation abilities. For the gait studies, lower extremity Fugl-Meyer (LEFM) [27•] scores ranged from 15 to 28 out of 34 points. However, there was great variability in motor severity within studies (standard deviations for three of these studies were larger than 12 points). One study split its sample into moderately and mildly impaired groups, with more impaired subjects making larger magnitude gains with both VR and control interventions [18•]. While better characterized than cognition, the stratification of motor severity in future studies will further aid in selective application of the technology.

The hardware interfaces used for both UL and walking systems were either robotic or motion sensing systems. Five of the eight UL studies, and one of the two walking studies, used a motion-sensing interface, while the remaining studies reported work with robotic systems. Neither interface appeared to yield superior motor outcomes. The addition of the sense of touch or haptics was included both UL and gait studies. Four UL systems were enhanced with haptics, three were purely visually based, 


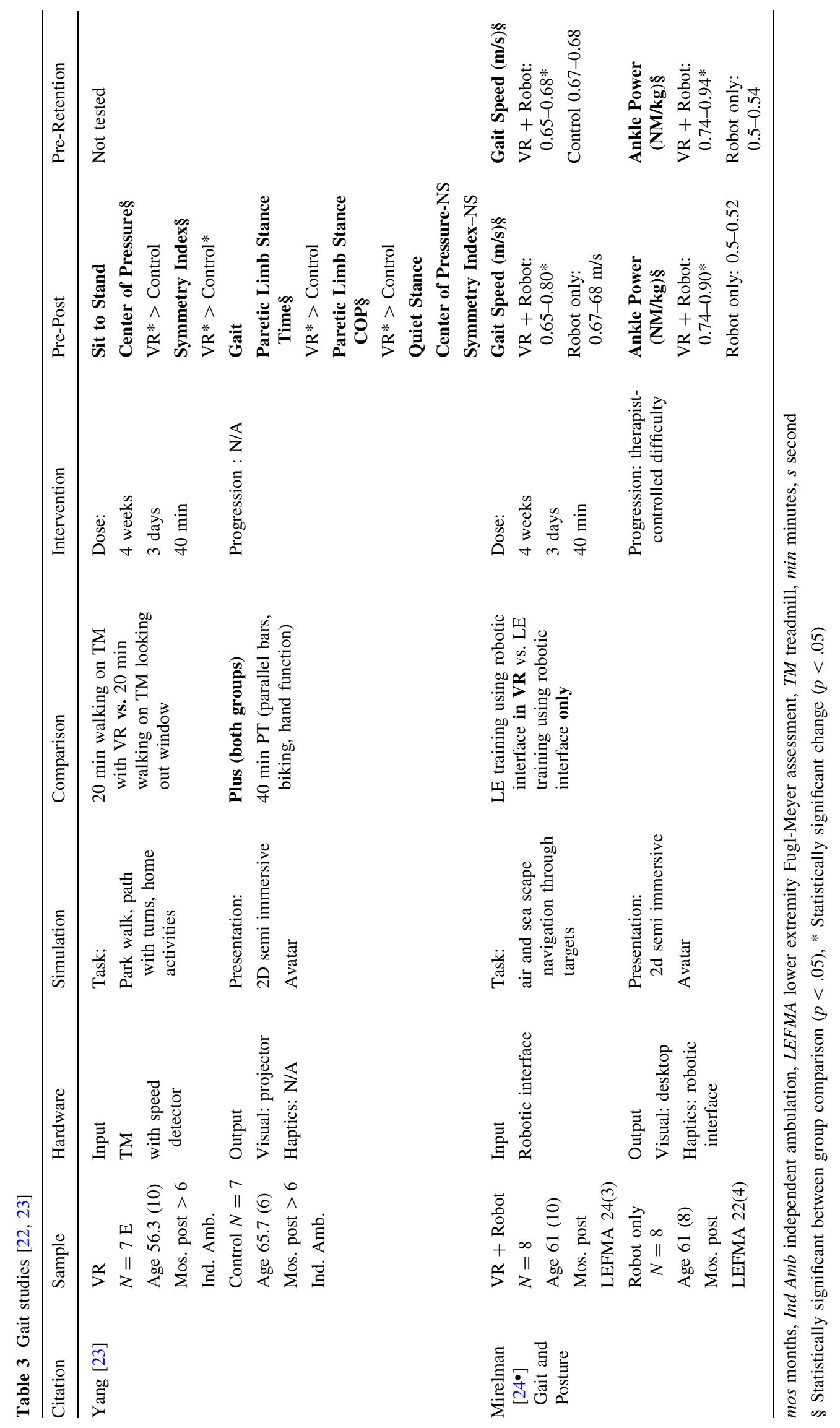


and one had a mixed reality where subjects interacted with real world objects. Controlled comparisons of interventions providing haptic feedback with similarly presented virtual interventions without haptic feedback were tested in two UL $[21 \bullet \bullet, 22]$ studies summarized in this article. The UL studies identified a small additive effect in terms of better real world activity level outcomes in subjects performing interventions with haptic feedback. The current literature does not point to robust clinically meaningful activity level outcomes for haptics added to VR systems.

Simulations for both the UL and walking were evenly grouped into two categories: replication of real world tasks such as reaching [18•] and walking [23], or game-based tasks, such as flying a plane with the impaired effector [24•]. For the UL, reaching tasks were performed by retrieving or transporting virtual objects to shelves and or table tops in virtual environments (VEs) that progressed from simple (a shelf) to complex (a supermarket) [17]. The games involved intercepting moving objects [15••, 21••], piloting an avatar through a VE [19], or sport simulations that required interaction with a ball [17]. For walking recovery, simulations were delivered in a variety of environments and similarly were divided into two groups: walking tasks that mimicked the trained tasks [23], and navigation simulations that promoted use of the lower extremities [24•].

Reaching simulations consistently improved reaching abilities [18•, 22], while game-based UL interventions produced less specific outcomes. In the gait studies, there were interesting findings, in that the game-based task coupled with a robotic interface showed a specific transfer of training to the trained distal effector as well as the task of walking [24•], while the walking simulation improved balance but not walking [23]. Task-specific training yielded superior results in the UL studies, but not in the gait studies.

As expected, visual and auditory feedback were presented by all of the systems with the visual presentation in either 2D or 3D. Most UL systems (six out of eight) were in $3 \mathrm{D}$, whereas both gait studies were in $2 \mathrm{D}$. The effect of the type of visual stimuli presentation in virtual rehabilitation is frequently discussed in a steadily growing literature. The majority of these studies compare UL activities performed in VEs with similar activities performed in real world environments [30], or compare immersive (3D) systems to flat-screen 2D systems [31, 32]. These two lines of inquiry identify differences in the kinematics of virtual UL and real world activities and differences in the kinematics of immersive and non-immersive virtual UL activities. Subramanian et al. [18•] identified differences reaching strategies developed in response to real world and virtual UE interventions. However, while the fidelity of movement is enhanced with 3D immersive systems, they are not superior to $2 \mathrm{D}$ non-immersive systems in promoting real world activity level motor outcomes.

Descriptions of feedback (KP and KR) were highly variable. There was great detail and attention to it in some papers [18•, 20•], reference to previous publications $[15 \bullet \bullet, 16,21 \bullet \bullet$, and no specific mention in others $[17,19$, 22]. Augmented feedback using KR was the predominant form of feedback provided in the UL studies. Exceptions to this were KP information on hand aperture [19] and trunk substitutions [18•]. Feedback also was distorted [20•] by providing error augmentation for arm trajectories. For the gait studies, feedback either was either not well described [23] or combined both KP (force generation and movement excursion of the foot) and KR [24•]. In the articles reviewed in this paper, there was no experimental manipulation of feedback making it difficult to determine whether combining KP and KR is superior to the provision of KR alone.

The frequency, duration and distribution of training in VR differed in some respects for the UL and gait studies. For both the UL and gait studies, training was typically distributed over a week. The duration on average tended to be approximately 4 weeks, but was 12 weeks for some UL studies. Total training time also was longer for the UL studies, with an average of $10.5 \mathrm{~h}$ of training compared to $7.5 \mathrm{~h}$ in the gait studies. For both the UL and gait studies, the differences in training time can be partially explained by greater time required for the systems that integrated robotics with VR. In addition, the gait study that required longer training duration (720 min) was performed in sitting [23], compared to the shorter training period performed in standing (270 $\mathrm{min})$ [22].

Relationships between training dose and improvements in motor function emerged. UL studies supported a doseresponse relationship between increased training dose and larger improvements in motor function identified by previous authors [31]. This trend was similar to the LL/gait studies, in which a larger dose of lower limb movements produced better outcomes [26] than a smaller volume of VR enhanced gait training [25].

Some of the strongest evidence related to the effectiveness of non-technology-based rehabilitation interventions suggests that outcomes are related to the dose of the intervention [32-35]. Training dose can be quantified as total treatment time or repetitions performed. This review only identified a single study in which training time was manipulated [19] experimentally, with better outcomes demonstrated by the group that trained in VR for a longer period of time. A large majority of the UL studies reviewed used a training time below the $16 \mathrm{~h}$ threshold dose associated with positive behavioral outcomes in the literature on non-automated rehabilitation of the upper extremity in 
persons with sub-acute stroke [35]. It is not clear if training doses in VR will compare to real world training doses.

The number of repetitions performed during training is another important element of training dose. Only one UL [18•] and one gait study [24•] controlled for the number of repetitions performed by the two treatment groups. When controlling for repetitions in the UL study, there were no differences between groups with respect to impairment and activity-level motor outcomes. By contrast, in the gait study, while repetitions were comparable between groups, training duration was greater for the VR group compared to the non-VR group. The authors speculated that the cognitive requirements to complete the same number of movements in VR accounted for the increased duration of training [24•]. These findings raise the question of which dose parameters (duration and or repetitions) should be controlled for to allow dose-matched comparisons between real world and VR training.

Treatment progression was achieved with software algorithms and clinician-tester input. More UL studies (5 out of 8) used an algorithm for progression. For UL studies in the absence of an algorithm, treatment progression was not well described. Output parameters included assistance provided by the algorithm [19, 22] and movement error augmentation [19]. The most sophisticated algorithm used movement parameters such as smoothness of sub-movements to shape the motor behavior and progress the therapy [15••, 21••]. For gait studies, progression of treatment either was not reported [23] or based upon performance accuracy and tester observation [24•]. It appears that robotic interfaces have the advantage of using sophisticated algorithms for treatment customization and progression. Whether this benefit merits the cost is still not clear.

Outcomes of VR training were identified at all levels of the ICF continuum with a predominance of body-structure and activity measures. Several of the UL studies demonstrated statistically significant improvements at the body

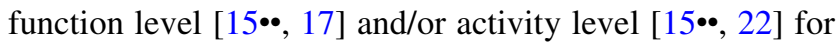
both VR groups and dose-matched controls. None of these studies that compared group outcomes demonstrated significant time-group interactions. It is also interesting to note that the largest magnitude improvements were reported by the study that examined subjects in the acute stage of recovery [21••], while the smallest magnitude changes were reported for the study with the smallest total treatment dose. Beyond this clear observation, patterns for dose-response or acuity levels impacting outcomes were not apparent. Only one study demonstrated statistically significant improvements in a participation level measure [17]. This study utilized the MAL, a self-report comprehensive UL measure. Other articles not identifying statistically significant improvements, considered the Barthel Index, a global measure of ADL function [21••] and the SIS hand sub-scale a measure examining finger based activities [19].

For both gait studies, there was a transfer of training to either or both body structure and activity measures. The VR training using a treadmill was not superior to real world training for walking, but did improve balance [23]. By contrast, the robotic-interfaced training in sitting coupled with VR [24•] was significantly better than the robot-alone condition for both body function measures, such as kinetics at push-off and activity measures such as walking velocity. For the two studies included in this review, there was no standardization of measures, such as the walking velocity and endurance, across studies, making comparison of outcomes difficult. This is in part explained because both studies aimed to elucidate some of the biomechanical explanations for changes in motor behavior, rather than measure the activity of walking.

\section{Conclusion}

While the quantity of RCT's examining VR for stroke rehabilitation is growing, there remain unresolved questions about the technology, clinical characteristics, and practical concerns that will affect translation of VR rehabilitation into practice. We summarize them here in an effort to frame relevant questions for the field.

Unresolved questions related to the technology:

- Is it necessary to utilize haptic interfaces to provide tactile feedback and interactive forces, or we can achieve similar transfer of training by using mixed reality systems?

- Are semi-immersive 2D systems as efficacious as immersive systems in reducing impairments and promoting activity? Does the use of non-immersive systems promote compensations?

- Do software-controlled algorithms provide greater speed and fidelity of exercise progression? Are they superior to expert clinical decision-making? Can they be combined with clinician-decision making?

Clinical application questions related to participant selection and specificity of training:

- What are the minimal cognitive and perceptual requirements identified by standardized assessments to use virtual environments for sensorimotor rehabilitation effectively?

- How will differences in motor severity, chronicity, and type of task interact with VR system capabilities and dose requirements?

Practical considerations

- Will the cost of the sophisticated technology (such a robots interfaced with the VE's) justify the benefits? 
We suggest that future studies should address both the efficacy of virtual rehabilitation interventions relative to the current standard of care, as well as comparing the efficacy and effectiveness of various approaches to presenting and delivering virtual interventions. In addition, while not the focus of this paper, studies that clarify the mechanisms underlying VR to stroke rehabilitation will complete our wish list. Comparative efficacy studies will enable us to answer the clinical and practical concerns, and the withinVR and mechanistic studies will aid in refining technology refinement, as well as elucidating the active ingredients in VR that will serve as a basis for stroke recovery.

Disclosure Dr. Deutsch is an inventor of virtual reality augmented systems. She presents CME on the topic of VR and Stroke.

\section{References}

Papers of particular interest, published recently, have been highlighted as:

- Of importance

•- Of major importance

1. Rizzo AA, et al. Analysis of assets for virtual reality in neuropsychology. Neuropsychol Rehabil. 2004;14:207-39.

2. Weiss PL, et al. Video capture virtual reality as a flexible and effective rehabilitation tool. J Neuroeng Rehabil. 2004;1(1):12.

3. Kimberley TJ, et al. Comparison of amounts and types of practice during rehabilitation for traumatic brain injury and stroke. J Rehabil Res Dev. 2010;47(9):851-62.

4. Adamovich SV, et al. Sensorimotor training in virtual reality: a review. Neurorehabilitation. 2009;25(1):29-44.

5. Sveistrup H. Motor rehabilitation using virtual reality. J Neuroeng Rehabil. 2004;1(1):10.

6. Saposnik G, Levin M. Outcome research Canada working, virtual reality in stroke rehabilitation: a meta-analysis and implications for clinicians. Stroke. 2011;42(5):1380-6.

7. - Laver KE, et al. Virtual reality for stroke rehabilitation. Cochrane Database Syst Rev. 2011;9:CD008349. This cochrane review identified eight UL and three gait studies, four global motor function, four activity re-training and one visual-perceptual training, in which VR was compared to the standard of care. Studies were performed primarily on individuals in the chronic phase post-stroke, in outpatient settings. Most of the systems were custom made virtual environments, with a two off-the-shelf game console. The primary findings were: limited evidence that the use of virtual reality and interactive video gaming may be beneficial in improving arm function and activities of daily living function when compared with the same dose of conventional therapy. There was insufficient evidence to reach conclusions about the effect on gait speed.

8. Holden MK. Virtual environments for motor rehabilitation: review. Cyberpsychol Behav. 2005;8(3):187-211.

9. Deutsch JE, et al. Post-stroke rehabilitation with the Rutgers ankle system: a case study. Presence Teleoper Virtual Environ. 2001;10(4):416-30.

10. Holden MK, et al. Virtual environment training improves motor performance in two stroke patients. J Neurol Phys Ther. 1998; 22(5): 179 .
11. Jaffe DL, et al. Stepping over obstacles to improve walking in individuals with poststroke hemiplegia. J Rehabil Res Dev. 2004;41(3A):283-92.

12. Levin MF, et al. Virtual reality environments to enhance upper limb functional recovery in patients with hemiparesis. Stud Health Technol Inform. 2009;145:94-108.

13. Weiss PL, et al. Video capture virtual reality: a decade of rehabilitation assessment and intervention. Phys Ther Rev. 2009;14(5):307-21.

14. Magill RA. Motor learning and control. 8th ed. Boston: McGrawHill; 2006.

15. $\bullet$ da Silva Cameirao M, et al. Virtual reality based rehabilitation speeds up functional recovery of the upper extremities after stroke: a randomized controlled pilot study in the acute phase of stroke using the rehabilitation gaming system. Restor Neurol Neurosci. 2011; 29(5):287-98. This paper is the most extensive study of virtual rehabilitation for persons in the early acute phase of recovery from stroke (less than thirty days) done to date. Multiple authors hypothesize that this phase of rehabilitation may be particularly amenable to virtual interventions.

16. Crosbie $\mathrm{JH}$, et al. Virtual reality in the rehabilitation of the arm after hemiplegic stroke: a randomized controlled pilot study. Clin Rehabil. 2012;26(9):798-806.

17. Levin MF, et al. Virtual reality versus conventional treatment of reaching ability in chronic stroke: clinical feasibility study. Neurol Ther. 2012;1(3):1-15.

18. - Subramanian SK, et al. Arm motor recovery using a virtual reality intervention in chronic stroke: randomized controlled trial. Neurorehabil Neural Repair. 2013;27(1):13-23. This paper presents a particularly well-controlled comparison of VR and non$V R$ based interventions. It also highlights the issue that different training modalities may elicit adaptations resulting in differing patterns of change movement patterns that do not translate to differing levels of improvement in clinical tests of motor function.

19. Hwang CH, Seong JW, Son D-S. Individual finger synchronized robot-assisted hand rehabilitation in subacute to chronic stroke: a prospective randomized clinical trial of efficacy. Clin Rehabil. 2012;26(8):696-704.

20. - Abdollahi F, et al. Arm control recovery enhanced by error augmentation. In: IEEE Int Conf Rehabil Robot.; 2011. p. 1-6. This paper describes the responses of persons with stroke to error augmentation, an approach that cannot be duplicated in traditionally presented rehabilitation environments.

21. • Cameirao MS, et al. The combined impact of virtual reality neurorehabilitation and its interfaces on upper extremity functional recovery in patients with chronic stroke. Stroke. 2012;43(10):2720-8. This paper provides a controlled comparison of virtual rehabilitation presented in isolation, augmented by an exoskeleton robot and the same intervention interfaced with a simpler haptic interface. It is the first study that considers the additive effects of robotics on virtual rehabilitation. This consideration will have impact on the development of future virtual rehabilitation systems, the economics of virtual rehabilitation and the translation of lab based systems to tele-rehabilitation and clinical environments.

22. Connelly L, et al. A pneumatic glove and immersive virtual reality environment for hand rehabilitative training after stroke. IEEE Trans Neural Syst Rehabil Eng. 2010;18(5):551-9.

23. Yang $\mathrm{S}$, et al. Improving balance skills in patients who had stroke through virtual reality treadmill training. Am J Phys Med Rehabil. 2011;90(12):969-78.

24. - Mirelman A, et al. Effects of virtual reality training on gait biomechanics of individuals post-stroke. Gait Posture. 2010;31(4):433-7. Companion to paper published in stroke in which VR coupled with a LE robot interface had superior outcomes for gait speed (measured in the lab and community), gait 
endurance (6 min walk) when compared to training with a repetition matched dose using the LE robot alone. In this paper the biomechanics describing the groups indicated that push-off during gait was the primary between group outcome. This supports specificity of training the distal effector. However, it raises the question of why task-based (navigation in a VE) training transferred to improved walking in the real world (in the absence of task specific training of walking).

25. Gaubert CS, Mockett SP. Inter-rater reliability of the Nottingham method of stereognosis assessment. Clin Rehabil. 2000;14(2):153-9.

26. Hodkinson HM. Evaluation of a mental test score for assessment of mental impairment in the elderly. Age Ageing. 1972;1(4): 233-8.

27. - Mirelman A, et al. Virtual reality for gait training: can it induce motor learning to enhance complex walking and reduce fall risk in patients with Parkinson's disease? J Gerontol A Biol Sci Med Sci. 2011;66(2):234-40. Paper explicitly illustrates the integration of cognitive and motor elements into VR training.

28. Bart O, et al. Using video-capture virtual reality for children with acquired brain injury. Disabil Rehabils. 2011;33(17-18):157986.
29. Fugl-Meyer AR, et al. The post-stroke hemiplegic patient. 1. A method for evaluation of physical performance. Scand J Rehabil Med. 1975;7(1):13-31.

30. Knaut LA, et al. Kinematics of pointing movements made in a virtual versus a physical 3-dimensional environment in healthy and stroke subjects. Arch Phys Med Rehabil. 2009;90(5):793802.

31. Subramanian SK, Levin MF. Viewing medium affects arm motor performance in 3D virtual environments. J Neuroeng Rehabil. 2011;8:36.

32. Henderson A, Korner-Bitensky N, Levin M. Virtual reality in stroke rehabilitation: a systematic review of its effectiveness for upper limb motor recovery. Top Stroke Rehabil. 2007;14(2): 52-61.

33. Kwakkel G. Impact of intensity of practice after stroke: issues for consideration. Disabil Rehabil. 2006;28(13-14):823-30.

34. Kwakkel G, et al. Effects of augmented exercise therapy time after stroke: a meta-analysis. Stroke. 2004;35(11):2529-39.

35. Kwakkel G, Kollen B, Twisk J. Impact of time on improvement of outcome after stroke. Stroke. 2006;37(9):2348-53. 